\title{
The Role of Sensory Network Dynamics in Generating a Motor Program
}

\author{
Rafael Levi, ${ }^{1}$ Pablo Varona, ${ }^{1,2}$ Yuri I. Arshavsky, ${ }^{1}$ Mikhail I. Rabinovich, ${ }^{1}$ and Allen I. Selverston ${ }^{1}$ \\ ${ }^{1}$ Institute for Nonlinear Science, University of California, San Diego, La Jolla, California 92093-0402, and ²Grupo de Neurocomputación Biológica, Escuela \\ Politécnica Superior, Universidad Autónoma de Madrid, 28049 Madrid, Spain
}

\begin{abstract}
Sensory input plays a major role in controlling motor responses during most behavioral tasks. The vestibular organs in the marine mollusk Clione, the statocysts, react to the external environment and continuously adjust the tail and wing motor neurons to keep the animal oriented vertically. However, we suggested previously that during hunting behavior, the intrinsic dynamics of the statocyst network produce a spatiotemporal pattern that may control the motor system independently of environmental cues. Once the response is triggered externally, the collective activation of the statocyst neurons produces a complex sequential signal. In the behavioral context of hunting, such network dynamics may be the main determinant of an intricate spatial behavior. Here, we show that (1) during fictive hunting, the population activity of the statocyst receptors is correlated positively with wing and tail motor output suggesting causality, (2) that fictive hunting can be evoked by electrical stimulation of the statocyst network, and (3) that removal of even a few individual statocyst receptors critically changes the fictive hunting motor pattern. These results indicate that the intrinsic dynamics of a sensory network, even without its normal cues, can organize a motor program vital for the survival of the animal.
\end{abstract}

Key words: Clione limacina; motor program; spatiotemporal pattern; hunting behavior; sensory organ; statocyst; motor coordination

\section{Introduction}

The dynamics of sensory receptor networks are likely to play a role in sensory motor transformation. Numerous examples exist of systems in which the transformation from sensory inputs to motor outputs has been studied (Lee et al., 1988; Schwartz et al., 1988; Fortier et al., 1989; Masino and Knudsen, 1990; Andersen et al., 1993; Britten et al., 1996). The clearest examples are found in invertebrate systems in which the different processing stages between input and output are more directly accessible (Lewis and Kristan, 1998; Levi and Camhi, 2000; Beenhakker and Nusbaum, 2004). Normally, the role of intrinsic dynamics cannot be distinguished from regular sensory coding functions. In certain conditions, however, the sensory intrinsic dynamics may be revealed when it overcomes its normal physiological role. For example, it is believed that motion sickness and optical illusions are the result of a normal sensory system functioning abnormally. Another way to reveal the dynamical properties of the nervous system is by looking at extreme examples of animal behavior. We have suggested previously that statocysts in the mollusk Clione also have the capability of independently generating a motor program (Levi et al., 2004).

Clione is a planktonic mollusk that swims by rhythmic movements of a pair of wings (Wns). During swimming, Clione ac-

Received March 28, 2005; revised Aug. 30, 2005; accepted Sept. 15, 2005.

This work was supported by National Institutes of Health Grant 7R01-NS-38022, National Science Foundation Grant EIA-0130708, Fundación BBVA, and MEC BFI2003-07276. We thank Danielle Nichols and the field services of the 0cean Science Center, Memorial University of Newfoundland for their administrative and logistic help.

Correspondence should be addressed to Rafael Levi, Institute for Nonlinear Science, University of California, San Diego, 9500 Gilman Drive, La Jolla, CA 92093. E-mail:rlevi@ucsd.edu.

DOI:10.1523/JNEUROSCI.2249-05.2005

Copyright $\odot 2005$ Society for Neuroscience $\quad$ 0270-6474/05/259807-09\$15.00/0 tively maintains a head-up orientation (Arshavsky et al., 1985; Satterlie, 1985; Deliagina et al., 1998, 1999, 2000), under the control of signals from the statocysts (Panchin et al., 1995b; Deliagina et al., 1998, 1999, 2000). A deviation from the vertical orientation evokes compensatory changes in wing and tail motions. Clione is a predator that feeds exclusively on a related mollusk (Lalli, 1970). With an undeveloped visual system, it uses relatively nondirectional chemosensory information to trigger hunting behavior, which consists of fast swimming in continuously changing loops.

Previous work has shown that the statocysts are necessary for the production of hunting behavior and that the motor output produces dynamics that are consistent with output generated by a winnerless competition model of the statocyst (Varona et al., 2002; Levi et al., 2004). However, because we were unable to record or manipulate more than one statocyst receptor cell (SRC) at a time, it remained unclear how the output of a population of SRCs corresponds to the actual motor output during hunting.

Here, we compare the pattern produced in the statocyst network and the pattern formed in the motor nerves to demonstrate quantitatively the direct correlation between these events. Because other ganglia are interpolated between the statocysts and the motoneurons, this is probably the only tractable method for establishing causality. Using a new technique to study the interactions between two spatiotemporal patterns without the explicit knowledge of the underlying processing, we show that statocyst receptors influence the temporal relationships between different wing and tail motoneurons. We show that stimulation of the statocyst network produces motor dynamics that correspond to fictive hunting. And finally, we extend our previous results to 
demonstrate that killing a small number of SRCs results in a substantial change to the motor output.

\section{Materials and Methods}

Electrophysiology. Preparations for electrophysiological experiments were made in ice-cold seawater to prevent excitation of nociceptive afferent fibers. The preparation, including cerebral, pedal, and abdominal ganglia with the tail and wing nerves, was pinned to a Sylgard-lined Petri dish as described previously (Levi et al., 2004). Extracellular recordings from nerves were made by using glass suction electrodes or stainless-steel electrodes. To monitor tail activity, we recorded from pedal nerves supplying tail muscles (Deliagina et al., 1999). Intracellular recordings were made using glass electrodes $(10 \mathrm{M} \Omega$ ) filled with $3 \mathrm{M} \mathrm{KCl}$. The signals were acquired with a Digidata board (Molecular Devices, Union City, CA) and stored for later analysis with Dataview (http://www.st-andrews.ac.uk/ $\sim$ wjh/dataview/). The spikes were sorted from the extracellular recordings in Dataview, using threshold and the spike template. Because there was little superposition in spike firing, we could typically sort four or five units in the statocyst and the motor nerves. To produce the instantaneous firing rate plots [the spike density function (SDF)], spike timing was convolved with a Gaussian function in Matlab (MathWorks, Natick, MA). For the normalized plots, each value was divided by the maximum value of the plot.

Stimulation of the statocyst nerve (STN) was performed en passant through the same suction electrode, and an AC amplifier (AM Systems, Carlsborg, WA) was used for recording. A $500 \mathrm{~ms}$ pulse was generated with a Master-8 (AMPI, Jerusalem, Israel) stimulator and fed into the amplifier. The stimulation was performed in two configurations. In the first configuration, the preparation was grounded, and activity in the recording electrodes was monitored continuously but the STN activity was not. In the second configuration, one of the inputs to the AC amplifier was used as a ground. The STN electrode was switched from stimulus mode (in which no recording was done in any nerve) to record mode (in which all electrodes were recording).

Cell photoablation was performed by methods described previously (Miller and Selverston, 1979). The neuron was impaled with electrodes filled with carboxyfluorescein dye (Sigma, St. Louis, MO). A negative current of $\sim 5 \mathrm{nA}$ was delivered for 10-20 min until the neuron was loaded with dye. The preparation was illuminated with light at $400-500$ $\mathrm{nm}$ for 20-30 min until the filled neuron died.

Fictive hunting behavior was induced by application of physostigmine as in the work by Arshavsky et al. (1993) and Levi et al. (2004). To achieve fictive hunting, the seawater covering the isolated nervous system was replaced by seawater containing $10^{-6} \mathrm{M}$ physostigmine.

Comparing spatiotemporal patterns. Typically fictive hunting was composed of a series of coordinated bursts in several nerves, which we called episodes. The beginning of an episode was the start of the first burst in any of the nerves. Several units in the STN or motor nerves acting together during an episode of hunting were defined as an ensemble. Typically, there were from 4 to 15 hunting episodes analyzed during a recording of 30-60 min. For each ensemble, the SDF of each identified unit was used as an axis to create the $\mathrm{N}$-dimensional representation of a response ( $N$ is the number of units in the ensemble). The result was a $\mathrm{N}$-dimensional trajectory that started and ended at the same point for each episode, because the firing rate of each neuron was the same before the beginning of the burst and when the burst terminated. As a measure of similarity between two trajectories, we calculated the minimal sum of Euclidian distances between corresponding points $x_{1}$ and $x_{2}$ of the trajectories, as follows:

$$
\left(\sum_{t=1}^{T} \sqrt{\sum_{i=1}^{N}\left(\left(x_{i 1}(t)-x_{i 2}(t+j)\right)^{2}\right.}\right),
$$

where the indexes 1 and 2 refer to the two trajectories (either STC or motor), index $i$ runs over the number of units, $t$ runs over the discretized time $(1 \ldots T)$, and the minimization was done over the trajectories shifted in time to account for the selection of the origin of the episodes and the correlation of major events slightly offset in activation. In this calculation, if $(t+j)>T$, then $x_{i 2}(t+j)=x_{i 2}(t+j-T)$; typical values of $j$ in the minimization were always close to 0 .

We repeated this calculation for each pair of episodes recorded in the STN and separately in the motor nerves. We then constructed correlation matrices for the STN trajectories and the corresponding motor trajectories. If there is a correlation between the SRC activity and the motor output, episodes that are similar in their SRC activity will also be similar in their motor activity and vice versa. For statistics, we used Pearson's correlations with a significance level of 0.05 .

Measuring correlated activity between tail and wing. The instantaneous firing rate (i.e., the SDF) was calculated for each unit. We used principal component (PC) analysis (PCA) to evaluate the results (Jolliffe, 1986). The PCA assigns new variables that maximize the variance in the data. Therefore, by replacing the original variable with the PCA, one can reduce the dimensionality (caused by redundancy in variables) of the system while preserving its structure. The PCA has been used for two purposes: first, to visualize multidimensional plots and second, to reduce the multidimensional data into a one-dimensional variable for the correlation analysis. For three-dimensional (3D) visualization, we simply took the SDF of typically four or five units and plotted their first three PCs. For analysis of correlation, we used only the first PC of each recording to calculate cross-correlations between activity in different nerves [wing and left and right tail ( $\mathrm{Tr}$ ) nerves]. The average percentage of the total variability of the motor signals explained by each of the principal components of the data shown in this paper is the following: first PC, $53 \pm$ 9\%; second PC, $24 \pm 4 \%$; third PC, $13 \pm 4 \%$.

\section{Results}

Clione hunting behavior is very different from regular swimming. It is characterized by a series of fast loops in varying planes produced by wing and tail motion (Lalli, 1970) to scan the surrounding space in search of prey. The hunting is presumably initiated by chemosensory and tactile receptors. Many cerebral interneurons are activated during hunting (Panchin et al., 1995a), and at least one pair of cerebral interneurons was found to activate the statocyst receptors (Arshavsky et al., 1993; Norekian and Satterlie, 1993). It is known that the control of wings during swimming is done through a wing central pattern generator located in the pedal ganglion (Arshavsky et al., 1985). Tail motoneurons are responsible for directional tail bending. Our unpublished behavioral observations indicate that there are correlated changes in the wing and tail motion. We have studied this correlation in detail during fictive hunting in in vitro preparations. Fictive hunting can be evoked experimentally by applying the acetylcholinesterase inhibitor physostigmine (Arshavsky et al., 1993). Application of physostigmine to a preparation of the isolated CNS evokes bouts of fictive hunting behavior [i.e., the excitation of motor systems (cerebral protractor tentacle motoneurons, the central pattern generator for swimming, and buccal protractor neurons) involved in hunting behavior]. In addition, physostigmine produces a reversal of the reaction to head nerve stimulation imitating the response to head stimulation in the intact animal during hunting. After physostigmine application, head nerve stimulation evokes strong excitation of both protractor tentacle motoneurons and the swimming central pattern generator instead of their inhibition, as observed before physostigmine application. Therefore, using physostigmine gave us the possibility of an electrophysiological analysis of the neural mechanisms underlying hunting search behavior.

\section{Characterization of individual motor unit activity}

The behavioral experiments show that hunting behavior takes place in bouts of fast swimming and looping. Similarly, fictive hunting is characterized by a series of episodes, of the same time scale, consisting of coordinated bursts in the motor nerves sepa- 
A

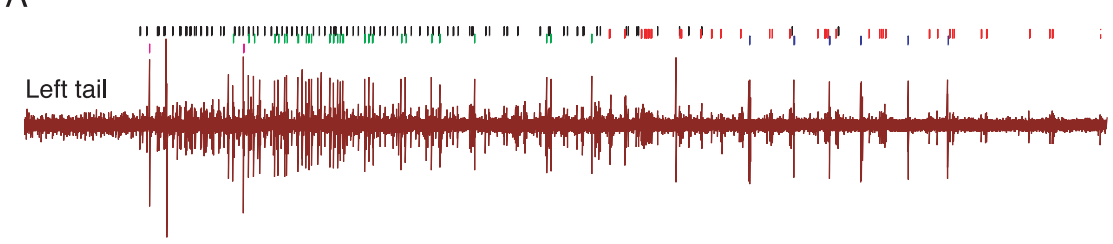

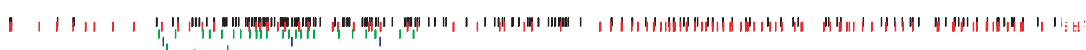

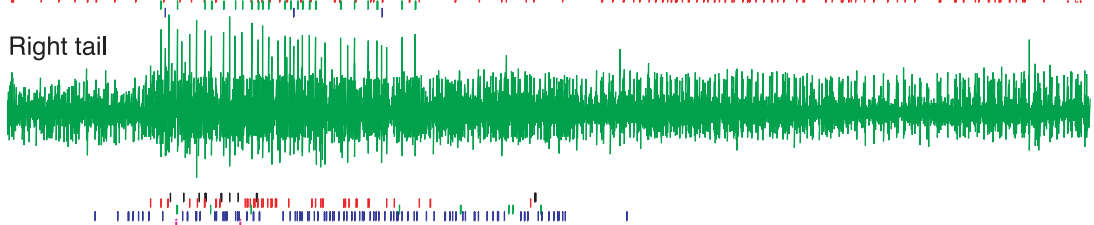

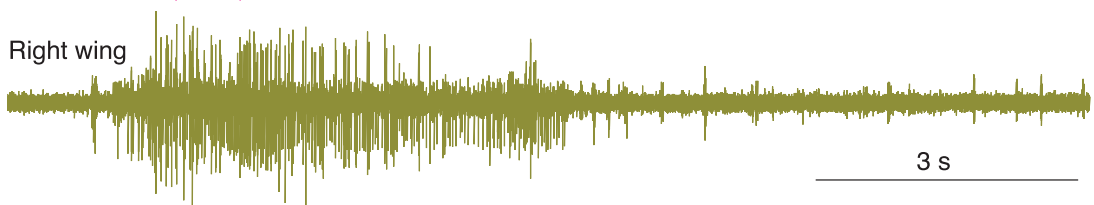

B

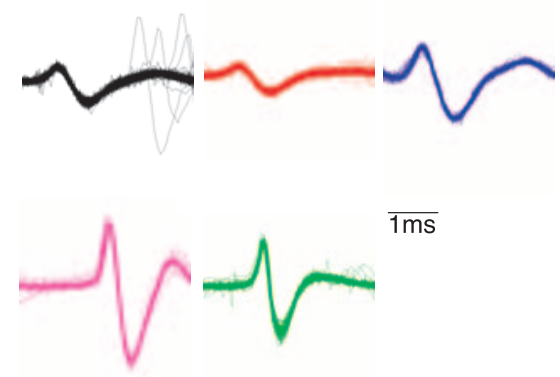

C

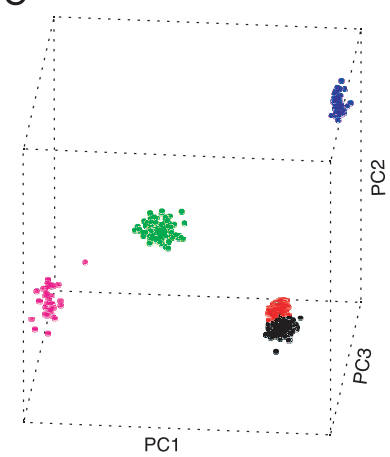

Figure 1. A typical motor response during an episode of fictive hunting. This episode is one of nine recorded in this trial. $\boldsymbol{A}$, Extracellular recordings from tail and wing motor nerves. Above each trace are color bars indicating the time of different colorcoded sorted units. $\boldsymbol{B}$, An overlay plot of the different sorted units in the left tail nerve in $\boldsymbol{A}$. In this panel, the sorting includes all the episodes in the trial. $\boldsymbol{C}, 3 \mathrm{D}$ plot of the clustering of the units in $\boldsymbol{B}$. Each spike is plotted according to its first three $P(s$. The color scheme is in accordance with $\boldsymbol{B}$. The number of spikes in each cluster is between 40 and 690 .

rated by quiescent periods. The reduced preparation that we used included the cerebral and pedal ganglia. The activity of both wing and tail was recorded from nerves originating in the pedal ganglia. Figure $1 A$ shows the activity of two tail nerves and one wing nerve during a hunting episode. A burst of activity starts and ends in several nerves in a time scale of seconds. The response was not simultaneous in all motor units. Rather, some units were activated early [Fig. $1 A$, black unit in left tail (Tl)] and others late (Fig. $1 A$, red unit in $\mathrm{Tl}$ ) in the episode. The beginning of an episode was defined as the start of the first burst in any of the nerves.

The spikes were sorted from the extracellular recordings using threshold and the spike template. Once the potential units were detected, they were sorted into clusters based on their three PCs. The average of each cluster was then used as a template, and the whole trace was searched for this template within an error of $10 \%$. The sorted spikes were verified manually as belonging to the same unit (Fig. $1 B, C$ ). Because there was little superposition in spike firing, we could typically sort four or five units in the statocyst and the motor nerves (Fig. $1 \mathrm{~A}$, small colored bars).

As the first step in the analysis of the wing and tail activity (i.e., the main effectors of Clione hunting behavior), we sought to characterize the activity of individual motor units. Figure 2 shows examples of recordings from different units in the tail and wing nerves in three separate experiments. Each panel $(A-C)$ represents one experiment from a different preparation and shows examples of three motor units during several fictive hunting episodes (rows within each panel). In each panel, the bursts were aligned according to the beginning of the hunting episodes to illustrate the variability between them. If one looks at the different episodes in the same panel, one can see that each unit has a characteristic behavior that repeats itself rather accurately. For example, in Figure $2 \mathrm{~A}$, right, the unit is bursting for a duration of $\sim 5 \mathrm{~s}$ with some small shifts in timing. As a result, the average of all the episodes (the bottom of each panel), in general, characterizes the firing pattern of each unit despite changes in the exact timing.

We found that throughout the experiments, the behavior could be classified into three characteristic time scales: units that fired short bursts relative to the duration of the episode, units that fired sporadic action potentials, and units that fired long bursts that include most of the episode duration. This characterization was observed in most of the recorded units in the experiments. Despite the consistency of the response, the exact timing of the activity was variable enough between bursts that the relative timing between units could change completely. In previous work (Levi et al., 2004), we showed that the timing of the activation does not change arbitrarily. Rather, the peak activity of each unit was separated from the others, and in many episodes, the sequential order of the activity was preserved. For that to happen, there has to be a preserved spatiotemporal relationship between units, which we sought to quantify.

\section{Similarity between SRC activity and motor output}

The statocysts are located on the dorsal side of the pedal ganglia. Each statocyst contains 9-11 receptor cells, the axons of which run into the cerebral ganglia through the STN and form two plexuses of neuropilar fibers in the medial areas of the ipsilateral and contralateral cerebral ganglia (Panchin et al., 1995b). SRCs are connected through electrical coupling and inhibitory synapses that are likely to be located in these regions of the cerebral ganglia. Although the preparation is stationary, during fictive hunting, the SRCs have a temporal activation pattern. As we showed above, combined with the results by Levi et al. (2004), it is impossible to correlate any single sensory unit with a motor pattern given the pattern is the outcome of a complex population activity. Nevertheless, it has been shown that the SRC activity is somehow associated with the activity in the motor nerves (Levi et al., 2004). One possibility is that if a population of neurons, instead of only one, is taken into account, such correlations can be found. In Figure 3, we show examples of recordings from the STN during hunting episodes in different animals. In each example, the spikes of four or five different receptors were sorted and translated into SDFs. A simultaneous recording from several 
SRCs shows that, with the exception of the red and blue units in Figure $3 B$, their peaks of activity do not coincide but demonstrate a sequential activation during the hunting episode. A similar spatiotemporal pattern with similar time scales has been described for the motor output to the wing and tail and modeled for the statocyst network by Levi et al. (2004). As was argued in the previous paper, this activity can be explained by a network of inhibitory interactions between statocyst receptors.

Because the activity of statocyst receptors was different in different episodes, we had the possibility to study the correlation between the statocyst activity and the motor output. To rigorously measure the correlation between sensory (SRCs) and motor (tail and wing) spatiotemporal patterns, we developed a method of quantifying differences in multidimensional spatiotemporal patterns. Figure 4 shows an experiment in which we recorded the activity of five units from one STN together with four units from one wing nerve and illustrates the analysis that we used. The figure shows two episodes (from the same animal and the same sorted units) in which the pattern in STN recording was similar in terms of the number, order, and timing of units being activated (Fig. 4A, two top panels). The third episode is notably different from the other two (Fig. $4 A$, bottom). The corresponding motor recordings (Fig. $4 B$ ) show that the top two patterns are similar to each other but different from the bottom panel. Although the similarity may be clear from this figure, additional statistical analysis was performed for more rigorous quantification. First, the similarity can be better visualized (see Materials and Methods) when looking at the trajectories in a phase portrait constructed with the three first principal components of five STN units (Fig. 4C) and four motor units (Fig. 4D). It is clear that the green and red trajectories (Fig. $4 A$, first two episodes) are close to each other and farther away from the blue trajectories (Fig. 4A, last episode). To quantify this, we have summed the Euclidean distances between SDFs in multidimensional space, where each dimension is the SDF for one motor or sensory unit. The distances for sensory and motor units were calculated separately, because they were composed of different dimensions (there was no apparent functional relationship between a sensory unit and a motor unit), and only combinations of distances in paired trajectories were compared (the distance between two sensory trajectories and two motor trajectories) within the same experiment.

To demonstrate a correlation between the STN and motor
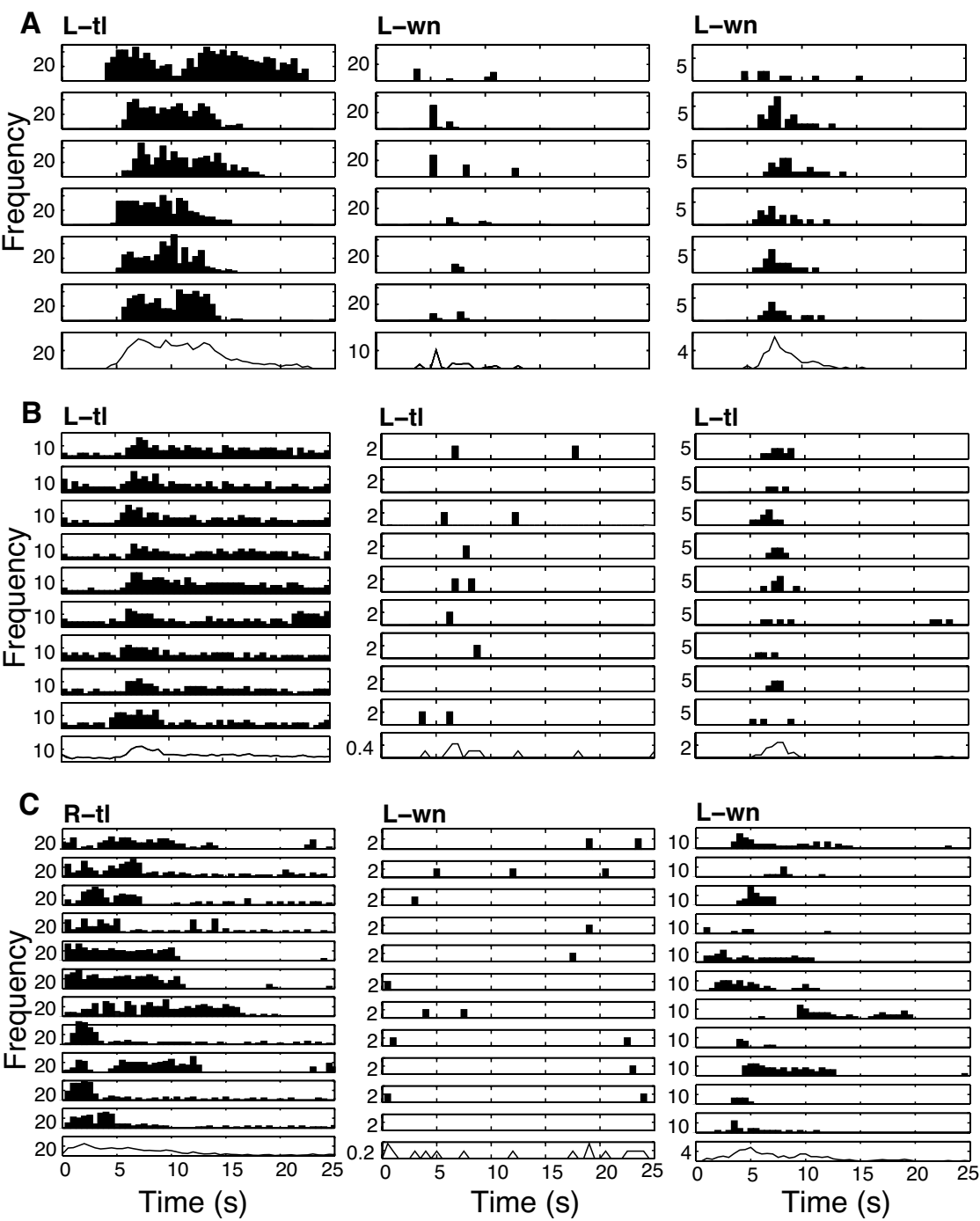

Figure 2. $\quad \boldsymbol{A}-\boldsymbol{C}$, Firing distribution of selected units from the tail and a wing nerves during different episodes of hunting from three experiments. Spike numbers were counted in $500 \mathrm{~ms}$ bins, and the activity was aligned to the beginning of the episode. The bottom of each panel is the summarized distribution calculated by averaging the corresponding bins in all the episodes above. The labels on the top left corner of each panel indicate the nerve that the unit was recorded from. The numbers on the $y$-axis indicate the frequency scale. $L$, Left; $R$, right; $T$ I, tail.
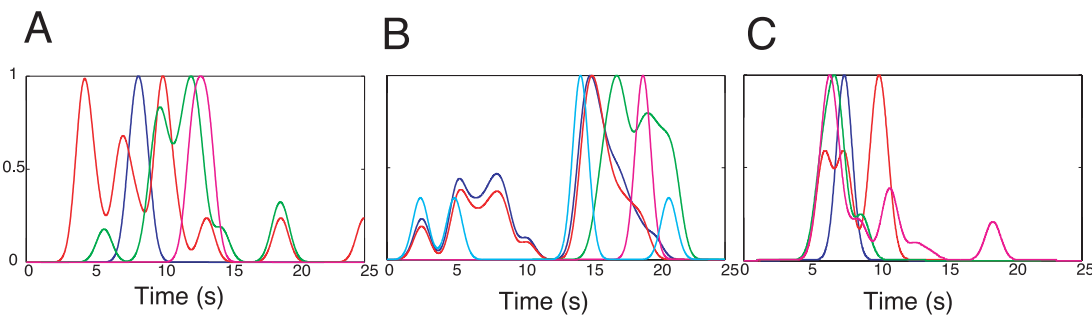

Figure 3. Firing pattern of SRC populations during hunting episodes. $\boldsymbol{A}-\boldsymbol{C}$, Three examples of SDFs recorded from the STN in different experiments. Each color represents an identified unit, the spikes of which were detected and translated into SDF (see Materials and Methods). Each experiment is different in terms of the recorded units and patterns. Although the activity of several units can overlap, the temporal activation shown in this figure is sequential in the sense that the peaks of unit activity are distributed over the duration of the hunting episode. 

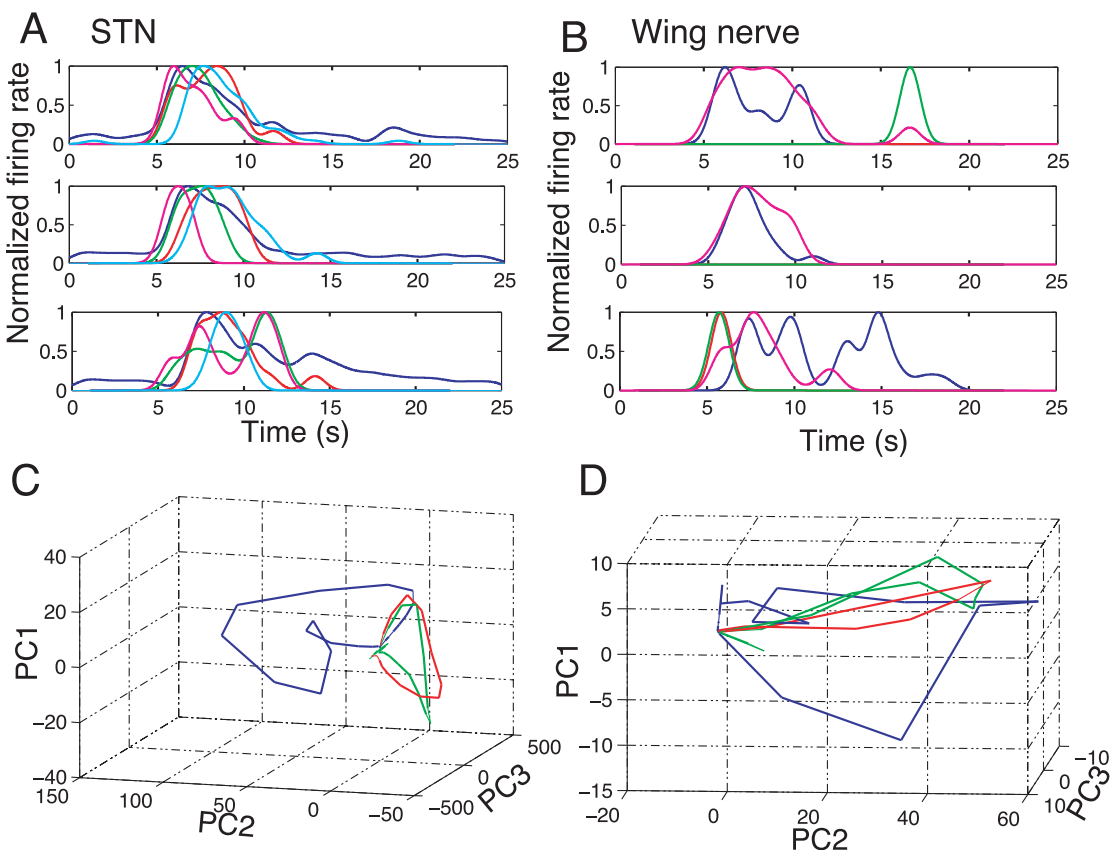

Figure 4. Relationship between spatiotemporal patterns during hunting episodes; similar spatiotemporal activity in the STN corresponds to a similar pattern in the motor nerves. $\boldsymbol{A}$, Examples of normalized SDFs from the STN during three episodes of fictive hunting. These recording were performed in the same animal, and five different SRC units were sorted. $\boldsymbol{B}$, SDF from wing motor nerves during the corresponding hunting episodes. $\boldsymbol{C}$, Three-dimensional representation of the responses in $\boldsymbol{A}$ using the first three PCs as axes (red, top panel in $\boldsymbol{A}$; green, middle panel in $\boldsymbol{A}$; blue, bottom panel in $\boldsymbol{A}$ ). $\boldsymbol{D}$, Three-dimensional representation of the responses in $\boldsymbol{B}$ using the first three $P(s$ as axes (colors as in $\boldsymbol{C}$ ).

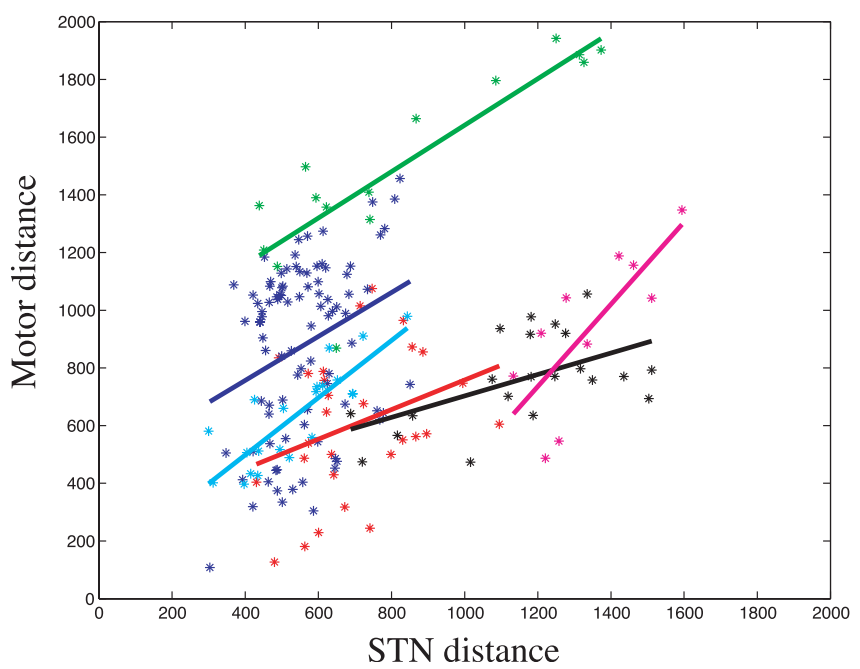

Figure 5. The correlation of distances between all STN episode patterns and motor episode patterns for all animals in which recording from the STN has been performed (arbitrary units). Lines are the linear fits of the corresponding experiment depicted in a different color. Only the experiment in black is not significantly correlated $(p=0.08)$.

Figure 5 shows all correlations between distances of the STN units and motor units in different animals. For example, the green plot shows the correlation between distances of five STN units and four motor units in seven consecutive episodes from the experiment in Figure 4 and its linear fit (each pair of distances was only used once in the analysis). This correlation is highly significant ( $p<0.01$; Pearson's correlation test), which means that episodes that are similar in their STN activity are also similar in their motor activity and vice versa. As can be seen in this figure, all linear fits have positive slopes, which indicates that as the stato- cyst distance increases the motor distance increases as well. Of a total of six experiments, we found significant correlation distances (Fig. 5) for five of them and one with a $p$ value of 0.08 .

\section{Stimulation of STC nerve}

The importance of the statocyst for hunting is illustrated by the fact that hunting behavior and fictive hunting cannot be initiated in statocystectomized animals (Levi et al., 2004). During fictive hunting, STN dynamics involving several receptors is recorded in the presence of a stationary sensory input. In the previous section, we showed the existence of a correlation between SRC and motor activity. We also showed examples in which individual SRC activity preceded the motor activity during fictive hunting (Levi et al., 2004). This evidence shows that the statocyst is an important player in the generation of the motor program but raises the question whether the statocyst can also initiate the program.

To test whether activation of SRCs can produce motor outputs similar to the ones observed during fictive hunting, we stimulated the STN as shown in Figure $6 \mathrm{~A}$. This small nerve contains mainly axons of the SRCs, and therefore nerve stimulation should produce action potentials that will activate the SRC network located proximally in the cerebral ganglia. After achieving a good recording from the nerve, we passed a short $(500 \mathrm{~ms})$ pulse of DC current while recording the tail and wing nerve activity. The intensity of the current was increased until a prolonged response in the motor nerve was observed. Typically, the voltage used was in the range of $1 \mathrm{~V}$, and the duration of the response became longer as the intensity was increased. The STN is too small to position both stimulating and recording electrodes. Therefore, as explained in Materials and Methods, in this experiment, we recorded continuously from the motor nerves but not from the STN. Figure $6, B$ and $C$, shows an example in which a short stimulation of the STN produces a long response in wing and tail motor nerves. The duration of the response was similar to a characteristic hunting episode duration (20 s; see above). During the response, the typical sequential activation of peak activity in different motor units was observed (compare Fig. 1A). This type of activity in the motor nerves has not been observed in any conditions other than fictive hunting. Similar results were observed in five other experiments. Repetitive stimulation, at intervals of $1 \mathrm{~min}$, resulted in a similar duration of the response but variable spatiotemporal patterns. Presumably, in these experiments, the stimulation activated several receptors simultaneously and induced a dynamic response in the SRC network without configurational changes attributable to the influence of neural or chemical inputs. In another set of experiments, we used the configuration that allowed recording from all nerves immediately after the stimulation, and in three experiments, we found that the STN activity pattern changed after the stimulation (Fig. $6 D$, fast firing rate in the STN trace). This change is prolonged and comparable with the prolonged activity pattern seen in the motor nerves. 


\section{Killing SRCs and its effect on the motor pattern}

If the signals from the SRC control the motor pattern, it would be expected that modifying the SRC network would have an effect on this pattern. To modify the SRC network, we photoablated two or three SRCs in a single statocyst and compared the fictive hunting motor output before and after the ablation. Hunting behavior was monitored by wing and tail nerve activity. Spikes were sorted from the extracellular nerve recordings as explained in Materials and Methods. The firing distribution of each motor cell was calculated. Each plot in Figure 7 is the summarized distribution calculated by averaging the corresponding bins in all the episodes (as the bottom panels in Fig. 2). The firing distribution reflects both the average firing rate and the reproducibility of the response.

Figure 7 shows the distribution of motoneuron firing during fictive hunting before and after ablation of three SRCs. A possible difficulty in interpreting the data arises from the long time that the ablation takes $(\sim 1 \mathrm{~h})$. This may result in the degradation of the system. However, in control experiments, we recorded fictive hunting for $\sim 60 \mathrm{~min}$ and observed no noticeable degradation in the unit activity. When three SRCs had been ablated, there were substantial changes in the firing pattern. Some units that fired in bursts before ablation started firing sporadically after the ablation (Fig. 7) (e.g., unit \#1 in $\mathrm{Tl}$ and unit \#3 in left Wn). Others increased their firing activity throughout the episode (e.g., unit \#5 in left Wn) or changed from nearly unimodal to clearly multimodal firing (e.g., units \#1 and \#5 in left Wn). Finally, previously silent units started firing (data not shown). Similar results were obtained in four other experiments.

These results indicate that the activity of few SRCs has an effect on the firing of individual motoneurons. To study population activity, we looked at the effect of SRC ablation on the ensemble dynamics of the wing and tail nerve activity during fictive hunting behavior for each experiment. To compare the multipleunit signals before and after ablation, we used PCA. First, we calculated the SDF for each unit. Then, we concatenated the episodes in each experiment (before and after) and subjected the multiple-unit SDF data to the PCA. The same transformation was applied to all the episodes in each treatment. The percentage of the total variability of the signals explained by the first principal component was $>50 \%$ for the analysis described below. Figure 8 shows the changes in time of the mean first PC of all episodes for three different nerves ( $\mathrm{Wn}, \mathrm{Tl}$, and right tail nerve) before (blue lines) and after (red lines) ablation. In this example, two of the nerves (wing and Tr) underwent major changes after the ablation, because there was a section of no overlap in the mean \pm SD (Fig. $8 A, C$ ). Figure 8 indicates that the progression of ensemble activity in two nerves critically changed after the killing of only three SRCs. Similar changes in ensemble activity were observed in 8 of 17 nerves recorded in six experiments.

The changes in coordination between different motor activities before and after ablation were analyzed by cross-correlation. We calculated the cross-correlation of the first PC between pairs of nerves. We computed the correlation between $\mathrm{Tl}$ and $\mathrm{Wn}, \mathrm{Tl}$ and $\mathrm{Tr}$, and $\mathrm{Tr}$ and Wn nerves. The motivation for this analysis was the possible participation of the statocyst signal in the coordination of wing and tail nerve activity expected from the participation of the statocysts in the generation of the motor program. Figure 9 indicates the existence of a high correlation (in absolute value) between all pairs before the ablation. After ablation, the correlations between the Tl-Wn and $\mathrm{Tr}-\mathrm{Tl}$ almost disappeared, whereas the $\mathrm{Tr}-\mathrm{Wn}$ correlation was somewhat reduced in absolute value but also changed sign. Reversal of the correlation sign means a phase change in activity before and after ablation. A positive correlation means that the activity has the tendency to be in phase with each other and vice versa. In the tail and wing cross-correlation, there were 6 of 11 changes in sign, and in the cross-correlation between the two tail nerve activities, there were four of five changes in sign. The conclusion was that changes in the network properties resulted in changes in the activity both in the individual motor units and in the ensemble spatiotemporal pattern. Despite the fact that we only killed a small portion of the total SRCs, the result was a substantial change in the motor spa- 


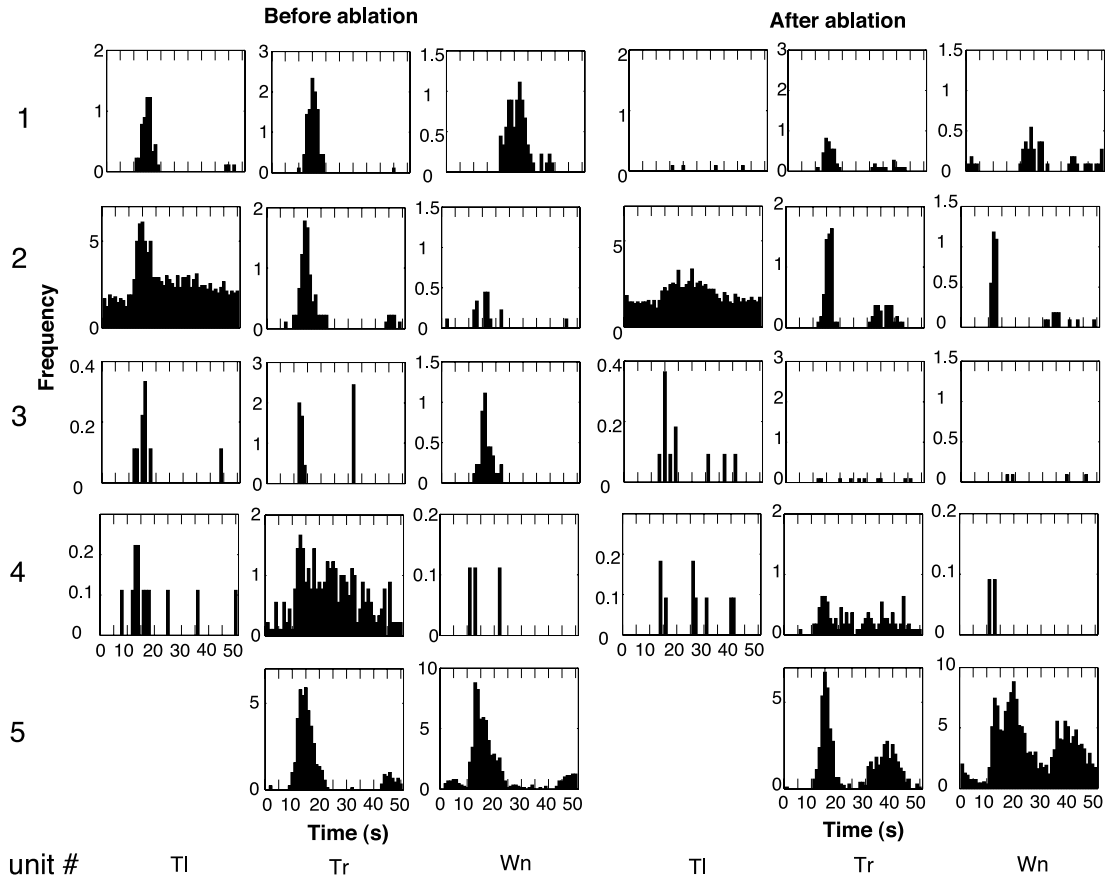

Figure 7. Firing distribution of units from the left and right tail nerves and a left wing nerve $(\mathrm{Wn})$ during fictive hunting before and after ablation of three SRCS. The burst were aligned to the beginning of the hunting episode. Note the change in the type of activity in some of the units.
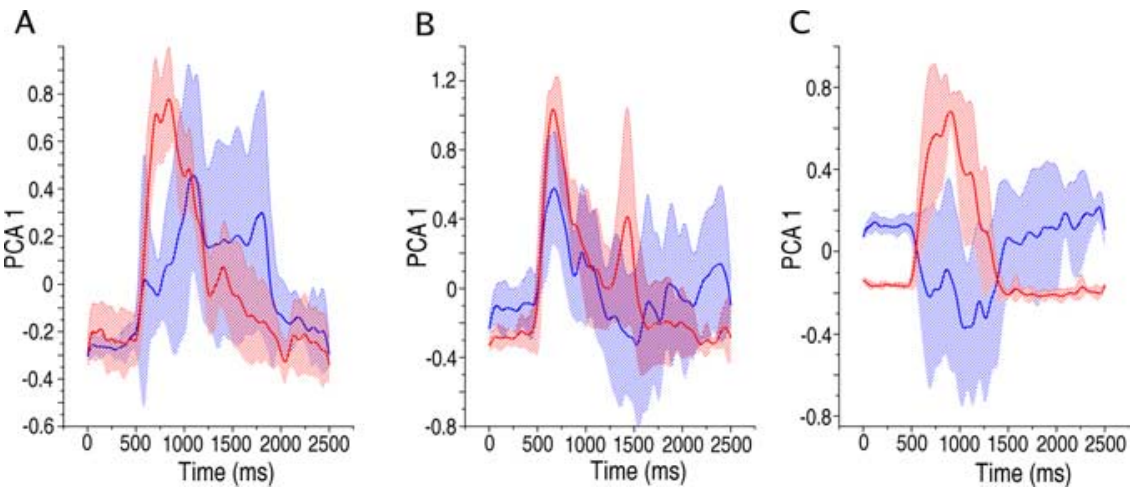

Figure 8. The average first $\mathrm{PC}$ before (blue) and after (red) ablation of three SRCs. $\boldsymbol{A}$, Wing; $\boldsymbol{B}$, left tail nerve; $\boldsymbol{C}$, right tail nerve. The shaded regions denote a range of \pm 1 SD from the mean $(n=7)$. Note regions without overlap in $\boldsymbol{A}$ and $\boldsymbol{C}$, which are regions that substantially changed.
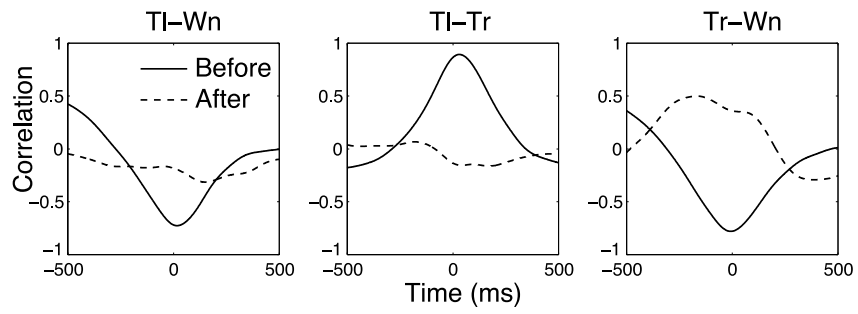

Figure 9. Change in cross-correlation of the first PC from Figure 7 before and after ablation of three SRCs for all possible nerve pairs. This cross-correlation was normalized so that the autocorrelations at 0 lag were equal to 1.

tiotemporal pattern. This was likely caused by the interaction of SRCs within the SRC network, because small perturbations in the network could qualitatively change the output attributable to the intrinsic network dynamics.

\section{Discussion}

Stimuli are encoded in sensory systems as spatiotemporal patterns and interpreted by the nervous system in a way that often generates a sequence of movements. Clione statocysts are sensory organs, the activity of which evokes a straightforward feedback motor response during normal swimming (i.e., to induce corrective motions in the wings and tail to achieve the preferred head-up position) (Deliagina et al., 1998, 1999, 2000). The results shown in this paper strongly support the idea of the dual functionality of a sensory network in two different behavioral tasks suggested by Levi et al. (2004). In particular, we showed conclusively that the statocysts are used to generate a spatially complex motor behavior. As was shown in the previous paper and here, only when looking at the population activity, it is possible to correlate the SRC dynamics with the motor output. Here, we were able to record and manipulate SRC populations confirming the fact that there is a relationship between the SRC dynamics and the motor program. The results presented also support a proposed model regarding the SRC network intrinsic dynamics in the work by Varona et al. (2002) and Levi et al. (2004). In this model, an external excitation to the SRC network triggered the generation of a switching dynamics caused by the winnerless competition among the receptors.

During hunting, the animal does not need to correct its body orientation relative to possible deviations from vertical. However, it has to produce fast and coordinated movements to capture its prey. This can be achieved by the same statocyst network using its intrinsic dynamics. Here, and in the work by Varona et al. (2002) and Levi et al. (2004), we showed that the statocyst network is able to produce complex spatiotemporal dynamics during hunting search behavior in contrast to the situation during routine swimming, in which only a few neurons are active. We also showed that the complex dynamics of the statocyst network during hunting governs, to a large extent, the motor output. A model showed that the statocyst network was able to produce the two kinds of dynamics observed in the experiments (Levi et al., 2004). In one state, it is a winner-take-all network, in which the statocyst receptors are active only when stimulated by a sensory cue. In the other state, the activity switches from one statocyst receptor to the other and produces complex dynamics. All it takes to transform the network from one state to the other is a broad excitation of the receptor network. Excitation in the Clione statocyst case may come from hunting interneurons (Arshavsky et al., 1993; Norekian and Satterlie, 1993), which brings the system out of the primarily inactive state. In the experiment shown in Figure 6, we showed that such excitation can also be evoked by directly stimulating the statocyst receptor axons. 
Sensory systems are supposed to transfer external information to be processed by the CNS. However, here, we have demonstrated an example in which a new spatiotemporal pattern is created by the dynamics of the sensory network, and this pattern is not a neural representation of the environmental state. In this extreme example, a sensory organ ceases to inform the nervous system about the environment and starts generating information independently. Less extreme situations may occur in the auditory (Wu and Oertel, 1986; Sobkowicz et al., 2003, 2004), vestibular (Ross, 1997; Uchino et al., 1999), visual (Dowling, 1970; Dacheux and Raviola, 1986; Wassle and Boycott, 1991; Masland and Raviola, 2000; Tsukamoto et al., 2001; Volgyi et al., 2002), and olfactory (Rall et al., 1966; Hinds, 1970; Kosaka and Hama, 1982; Kosaka et al., 2001; Laurent et al., 2001; Nezlin et al., 2003) systems in which elaborate interconnections and feedback exist. In such interconnected systems, the connectivity presumably can produce a more complex dynamics than the one necessary for the processing of the sensory signal but at the same time can be controlled by higher centers and altered, as the behavioral context requires it.

Although the spatiotemporal dynamics of the statocyst neurons during hunting is not generated by a sensory cue (i.e., it is not a representation of the environment), the signals are readily interpreted by the motor centers. The result is a motor pattern consisting of sequences of highly irregular but organized motor commands. It is reasonable to assume that the statocyst signals are processed as real sensory information, therefore ready to be used by the nervous system to produce, in turn, a functional motor pattern. The advantage of producing a spatiotemporal pattern in the statocyst is that these signals presumably already contain useful information for a coherent motor response (e.g., spatiotemporal cues for motor coordination). It is very likely that other parts of the nervous system undergo changes as well. These changes may have significant effects on the transformation from sensory to motor pattern and should be the subject of future study. However, our results show that the changes the statocyst undergoes are sufficient to explain the observed modification in the motor pattern and account for a substantial part of the control of the hunting behavior. Furthermore, these results suggest that the spatiotemporal organization of the statocyst signals can be crucial for the organization of behavior. In particular, the statocyst can be involved in coordination of activity during hunting. The correlation of motor nerves (in particular, wing and tail nerves) undergo significant changes after the ablation of statocyst receptor cells and thus support the hypothesis that the statocyst network indeed participates in specific tasks of the motor program such as motor coordination. In fact, the statocysts are programming the hunting behavior using the information about the presence of prey just as a triggering signal.

In the statocysts, the coding of sensory information is realized through a population code in which none of its members contains the full information about the stimuli. We have shown that the relationship between the statocyst signal and the motor output can be only seen when joint action is taken into account.

We have shown an example of a sensory organ dual functionality that depends on the behavioral context. The dual functionality can be achieved without long-term changes or neuromodulation. Rather, the functional reconfiguration is accomplished through transient excitation that brings the system into a different dynamical state. This kind of neural reconfiguration increases the versatility of the neural system and may be used by the animal to increase its behavioral repertoire by using existing neural structures and building on them.

\section{References}

Andersen RA, Snyder LH, Li CS, Stricanne B (1993) Coordinate transformations in the representation of spatial information. Curr Opin Neurobiol 3:171-176.

Arshavsky Y, Beloozerova GN, Orlovsky GN, Panchin YV, Pavlova GA (1985) Control of locomotion in marine mollusc Clione limicana III. On the origin of rhythmic activity. Exp Brain Res 58:273-284.

Arshavsky YI, Deliagina TG, Gamkrelidze GN, Orlovsky GN, Panchin YV, Popova LB (1993) Pharmacologically induced elements of the hunting and feeding behavior in the pteropod mollusk Clione limacina. II. Effects of physostigmine. J Neurophysiol 69:522-532.

Beenhakker MP, Nusbaum MP (2004) Mechanosensory activation of a motor circuit by coactivation of two projection neurons. J Neurosci 24:6741-6750

Britten KH, Newsome WT, Shadlen MN, Celebrini S, Movshon JA (1996) A relationship between behavioral choice and the visual responses of neurons in macaque MT. Vis Neurosci 13:87-100.

Dacheux RF, Raviola E (1986) The rod pathway in the rabbit retina: a depolarizing bipolar and amacrine cell. J Neurosci 6:331-345.

Deliagina TG, Arshavsky YI, Orlovsky GN (1998) Control of spatial orientation in a mollusc. Nature 393:172-175.

Deliagina TG, Orlovsky GN, Selverston AI, Arshavsky YI (1999) Neuronal mechanisms for the control of body orientation in Clione I. Spatial zones of activity of different neuron groups. J Neurophysiol 82:687-699.

Deliagina TG, Orlovsky GN, Selverston AI, Arshavsky YI (2000) Neuronal mechanisms for the control of body orientation in Clione II. Modifications in the activity of postural control system. J Neurophysiol 83:367-373.

Dowling JE (1970) Organization of vertebrate retinas. Invest Ophthalmol 9:655-680.

Fortier PA, Kalaska JF, Smith AM (1989) Cerebellar neuronal activity related to whole-arm reaching movements in the monkey. J Neurophysiol 62:198-211.

Hinds JW (1970) Reciprocal and serial dendrodendritic synapses in the glomerular layer of the rat olfactory bulb. Brain Res 17:530-534.

Jolliffe IT (1986) Principal component analysis. New York: Springer.

Kosaka K, Aika Y, Toida K, Kosaka T (2001) Structure of intraglomerular dendritic tufts of mitral cells and their contacts with olfactory nerve terminals and calbindin-immunoreactive type 2 periglomerular neurons. J Comp Neurol 440:219-235.

Kosaka T, Hama K (1982) Synaptic organization in the teleost olfactory bulb. J Physiol (Paris) 78:707-719.

Lalli CM (1970) Structure and function of the buccal apparatus of Clione limacina (Phipps) with a review of feeding in gymnosomatous pteropods. J Exp Mar Biol Ecol 4:101-118.

Laurent G, Stopfer M, Friedrich RW, Rabinovich MI, Volkovskii A, Abarbanel HD (2001) Odor encoding as an active, dynamical process: experiments, computation, and theory. Annu Rev Neurosci 24:263-297.

Lee C, Rohrer WH, Sparks DL (1988) Population coding of saccadic eye movements by neurons in the superior colliculus. Nature 332:357-360.

Levi R, Camhi JM (2000) Population vector coding by the giant interneurons of the cockroach. J Neurosci 20:3822-3829.

Levi R, Varona P, Arshavsky YI, Rabinovich MI, Selverston AI (2004) Dual sensory-motor function for a molluskan statocyst network. J Neurophysiol 91:336-345.

Lewis JE, Kristan Jr WB (1998) Quantitative analysis of a directed behavior in the medicinal leech: implications for organizing motor output. J Neurosci 18:1571-1582.

Masino T, Knudsen EI (1990) Horizontal and vertical components of head movement are controlled by distinct neural circuits in the barn owl. Nature 345:434-437.

Masland RH, Raviola E (2000) Confronting complexity: strategies for understanding the microcircuitry of the retina. Annu Rev Neurosci 23:249-284.

Miller JP, Selverston A (1979) Rapid killing of single neurons by irradiation of intracellularly injected dye. Science 206:702-704.

Nezlin LP, Heermann S, Schild D, Rossler W (2003) Organization of glomeruli in the main olfactory bulb of Xenopus laevis tadpoles. J Comp Neurol 464:257-268.

Norekian TP, Satterlie RA (1993) Cerebral neurons underlying prey capture movements in the pteropod mollusc, Clione limacina. I. Physiology, mor- 
phology. J Comp Physiol A Neuroethol Sens Neural Behav Physiol 172:153-169.

Panchin YV, Popova LB, Deliagina TG, Orlovsky GN, Arshavsky YI (1995a) Control of locomotion in marine mollusk Clione limacina. VIII. Cerebropedal neurons. J Neurophysiol 73:1912-1923.

Panchin YV, Arshavsky YI, Deliagina TG, Popova LB, Orlovsky GN (1995b) Control of locomotion in marine mollusk Clione limacina. IX. Neuronal mechanisms of spatial orientation. J Neurophysiol 73:1924-1937.

Rall W, Shepherd GM, Reese TS, Brightman MW (1966) Dendrodendritic synaptic pathway for inhibition in the olfactory bulb. Exp Neurol 14:44-56.

Ross MD (1997) Morphological evidence for local microcircuits in rat vestibular maculae. J Comp Neurol 379:333-346.

Satterlie RA (1985) Reciprocal inhibition and postinhibitory rebound produce reverberation in locomotor pattern generator. Science 229:402-404.

Schwartz AB, Kettner RE, Georgopoulos AP (1988) Primate motor cortex and free arm movements to visual targets in three-dimensional space. I. Relations between single cell discharge and direction of movement. J Neurosci 8:2913-2927.

Sobkowicz HM, Slapnick SM, August BK (2003) Reciprocal synapses be- tween inner hair cell spines and afferent dendrites in the organ of Corti of the mouse. Synapse 50:53-66.

Sobkowicz HM, August BK, Slapnick SM (2004) Synaptic arrangements between inner hair cells and tunnel fibers in the mouse cochlea. Synapse 52:299-315.

Tsukamoto Y, Morigiwa K, Ueda M, Sterling P (2001) Microcircuits for night vision in mouse retina. J Neurosci 21:8616-8623.

Uchino Y, Sato H, Kushiro K, Zakir M, Imagawa M, Ogawa Y, Katsuta M, Isu N (1999) Cross-striolar and commissural inhibition in the otolith system. Ann NY Acad Sci 871:162-172.

Varona P, Rabinovich MI, Selverston AI, Arshavsky YI (2002) Winnerless competition between sensory neurons generates chaos: a possible mechanism for molluscan hunting behavior. Chaos 12:672-677.

Volgyi B, Xin D, Bloomfield SA (2002) Feedback inhibition in the inner plexiform layer underlies the surround-mediated responses of AII amacrine cells in the mammalian retina. J Physiol (Lond) 539:603-614.

Wassle H, Boycott BB (1991) Functional architecture of the mammalian retina. Physiol Rev 71:447-480.

Wu S, Oertel D (1986) Inhibitory circuitry in the ventral cochlear nucleus is probably mediated by glycine. J Neurosci 6:2691-2706. 